\title{
The Effects of Cycle Temperature and Cycle Pressure Ratios on the Performance of an Irreversible Otto Cycle
}

\author{
Y. $\mathrm{UST}^{a, *}, \mathrm{~B} \cdot \mathrm{SAHIN}^{a}$ AND A. SAFA ${ }^{b}$ \\ ${ }^{a}$ Department of Naval Architecture and Marine Engineering, Yildiz Technical University \\ Besiktas, 34349, Istanbul, Turkey \\ ${ }^{b}$ Department of Marine Engineering Operations, Yildiz Technical University \\ Besiktas, 34349, Istanbul, Turkey
}

(Received January 11, 2011)

\begin{abstract}
This paper reports the thermodynamic optimization based on the maximum mean effective pressure, maximum power and maximum thermal efficiency criteria for an irreversible Otto heat engine model which includes internal irreversibility resulting from the adiabatic processes. The mean effective pressure, power output, and thermal efficiency are obtained by introducing the compression ratio, cycle temperature ratio, specific heat ratio and the compression and expansion efficiencies. Optimal performance and design parameters of the Otto cycle are obtained analytically for the maximum power and maximum thermal efficiency conditions and numerically for the maximum mean effective pressure conditions. The results at maximum mean effective pressure conditions are compared with those results obtained by using the maximum power and maximum thermal efficiency criteria. The effects of the cycle temperature ratio and cycle pressure ratio on the general and optimal performances are investigated.
\end{abstract}

PACS: 05.70.Ln, 88.05.-b, 88.20.td

\section{Introduction}

In the engines, working on the Otto cycle principle, it is considered that combustion takes place at a constant volume process. And also, as a reciprocating engine, besides combustion efficiency and heat loss, compression ratio is the major characteristics to determine the engine performance. Identifying the performance limits of thermal systems and optimizing thermodynamic processes and cycles including finite-time, finite-rate and finite-size constraints have been the subject of many thermodynamic modeling and optimization studies in physics and engineering literature [1-7].

Mozurkewich and Berry [8] incorporated the major loss-terms such as friction loss, heat leak and incomplete combustion in a simple model based on an air-standard cycle. Then using optimal control theory, the piston trajectory which yields maximum power output was computed.

Klein [9] considered the ideal Otto and Diesel cycles and compared their volumetric compression ratios and thermal efficiencies at maximum work per cycle conditions. He studied the effect of heat transfer through the cylinder wall on the work output of an Otto cycle assuming the heat transfer through the cylinder walls to be a linear function of the difference between the average gas and cylinder-wall temperatures during the isometric

* corresponding author; e-mail: yust@yildiz.edu.tr heat-addition process. $\mathrm{Wu}$ and Blank [10, 11] studied on the effect of combustion on a work-optimized endoreversible Otto cycle.

Hernandez et al. [12] have presented a simplified model for an irreversible Otto cycle with internally dissipative friction. The model considers non-instantaneous adiabatic processes and gives a central role to the full cycle time and piston velocity on the adiabatic branches.

Angulo-Brown et al. [13] proposed an irreversible simplified model for the air-standard Otto cycle, taking into account the finite times for heating and cooling processes. They also considered a lumped friction-like dissipation term representing global losses. They maximized the power output, efficiency and ecological function with respect to the compression ratio and showed that the optimum compression ratio values were in good agreement with those of standard values for practical Otto engines.

Chen et al. [14] derived the relation between net work output and efficiency of the air standard Otto cycle taking into consideration loss of heat transfer through the cylinder wall.

Ge et al. [15, 16] have taken into consideration the effect of variable specific heats on the performance characteristics of endoreversible and irreversible Otto cycles where variable specific heats of working fluid are linear functions of the temperature.

Ozsoysal [17] studied to characterize the amount of heat leakage as a percentage of the fuel's energy. He presented the valid ranges of heat transfer parameters of Otto and Diesel cycles in graphs and basic equations. 
Chen et al. [18] derived the power output and efficiency of the Otto cycle by establishing (using) an irreversible model. He has taken into account the effects of non-isentropic compression and expansion processes; finite-time processes and heat transfer loss through the cylinder walls.

Hou [19] analyzed the performances of the Otto and Atkinson cycles comparing with each other. He paid regard to heat transfer loss.

Ge et al. [20] analyzed the effects of internal reversibilities, heat transfer and friction losses on the performance of the irreversible Otto cycle. He calculated the non-linear relation between the specific heat of the working fluid and its temperature, friction loss according to the mean speed of the piston and determined the internal irreversibility by using the compression and expansion efficiencies.

Lin et al. [21] studied to characterize the effects of heat loss by percentage of the fuel's heat content, friction and variable specific heats of working fluid on the performance of the Otto cycle with constraint of maximum cycle temperature.

Gumus et al. [22] has performed a comparative performance analysis for a reversible Otto cycle based on three alternative performance criteria namely maximum power $(\mathrm{mp})$, maximum power density (mpd) and maximum efficient power (mep). They defined the power density criterion as the power per minimum specific volume in the cycle and the efficient power criterion as multiplication of the power by the efficiency of the Otto cycle. They have also found that the design parameters at mep conditions lead to more efficient engines than that at the mp condition and the mep criterion may have a significant power advantage compared with mpd criterion.

Ebrahimi [23] has also analyzed the effects of variable specific heat ratio on performance of an endoreversible Otto cycle. He derived the relations between the work output and the compression ratio, between the thermal efficiency and the compression ratio by using the finite time thermodynamics.

In this thermodynamics analysis, a comparative performance analysis is carried out for an irreversible Otto cycle based on three alternative performance criteria namely, maximum mean effective pressure (MEP), maximum power (MP) and maximum thermal efficiency (MEF) by considering the internal irreversibility resulting from the adiabatic processes. In this context, the general and optimal performance and design parameters under MEP, MP and MEF conditions are comparatively discussed. Additionally, the effects of cycle temperature ratio and cycle pressure ratio on the performance will also be examined.

\section{Performance optimization for an irreversible Otto cycle}

$P-V$ and $T-S$ diagrams of a standard irreversible Otto cycle with air is shown in Fig. 1. In the $T-S$ diagram, the

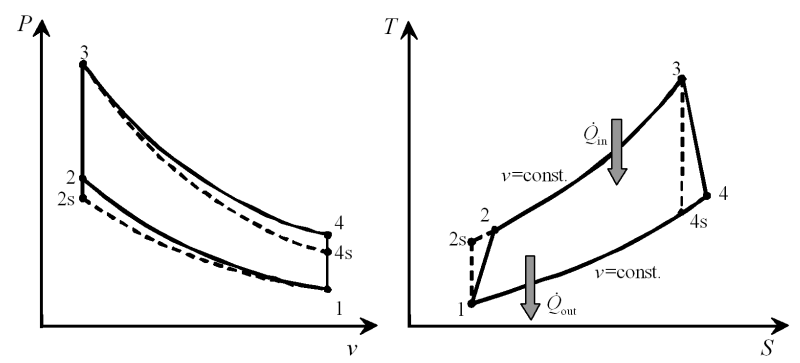

Fig. 1. $P-V$ and $T-S$ diagrams for the irreversible Otto cycle.

process $1-2 \mathrm{~s}$ is an isentropic compression, while the process 1-2 takes into account internal irreversibilities. The heat addition occurs in the constant volume process $2-3$. Process $3-4 \mathrm{~s}$ is an isentropic expansion, while the process 3-4 takes into account internal irreversibilities. A constant volume heat rejection process, $4-1$, completes the cycle. The heat added to the working fluid during the process $2-3$ is

$$
\dot{Q}_{\text {in }}=\dot{Q}_{23}=\dot{m} C_{V}\left(T_{3}-T_{2}\right)
$$

and the heat rejected by the working fluid during the process $4-1$ is

$$
\dot{Q}_{\text {out }}=\dot{Q}_{41}=\dot{m} C_{V}\left(T_{4}-T_{1}\right) \text {. }
$$

The power output of the cycle can be written in the form

$$
\dot{W}=\dot{Q}_{\text {in }}-\dot{Q}_{\text {out }}=\dot{m} C_{V}\left[\left(T_{3}-T_{2}\right)-\left(T_{4}-T_{1}\right)\right]
$$

and the thermal efficiency can be written as

$$
\eta=1-\frac{\dot{Q}_{\text {out }}}{\dot{Q}_{\text {in }}}=1-\frac{T_{4}-T_{1}}{T_{3}-T_{2}}
$$

where $\dot{m}$ is the cycle mass times the number of cycles per second, $C_{V}$ is the constant volume specific heat capacity and $T_{1}, T_{2}, T_{3}$ and $T_{4}$ are temperatures at states 1,2 , 3 , and 4 , respectively. The displacement volume of the cycle, $v_{\mathrm{d}}$, can be written as

$$
v_{\mathrm{d}}=v_{\max }-v_{\min }=v_{1}-v_{2} .
$$

The mean effective pressure defined as the ratio of power output to the displacement volume in the cycle then takes the form

$$
P_{\mathrm{ME}}=\dot{W} / v_{\mathrm{d}}=\frac{\dot{m} C_{V}\left[\left(T_{3}-T_{2}\right)-\left(T_{4}-T_{1}\right)\right]}{v_{1}-v_{2}} .
$$

The second law of thermodynamics requires that

$$
T_{2 S} T_{4 S}=T_{3} T_{1} \text {. }
$$

For the two adiabatic processes, the compression and expansion efficiencies [6]:

$$
\eta_{C}=\frac{T_{2 S}-T_{1}}{T_{2}-T_{1}}
$$

and

$$
\eta_{E}=\frac{T_{3}-T_{4}}{T_{3}-T_{4 S}}
$$

can be used to describe the irreversibility of the adiabatic 
processes. Using Eqs. (7), (8) and (9), we obtain

$$
T_{2} / T_{1}=\frac{\eta_{C}+\left(r^{(k-1)}-1\right)}{\eta_{C}}
$$

and

$$
T_{4} / T_{1}=\alpha\left[1-\eta_{E}\left(1-\frac{1}{r^{(k-1)}}\right)\right],
$$

where $k$ is the ratio of the specific heat capacities $\left(C_{P} / C_{V}\right)$ and the cycle temperature ratio $(\alpha)$ and compression ratio $(r)$ are defined as

$$
\begin{aligned}
& \alpha=T_{3} / T_{1} \\
& r=\frac{v_{\max }}{v_{\min }}=\frac{v_{1}}{v_{2}} .
\end{aligned}
$$

In terms of these parameters, it is easy to find the dimensionless power output, thermal efficiency, and dimensionless mean effective pressure by substituting Eqs. (10) and (11) into Eqs. (3), (4) and (6), respectively, as

$$
\begin{aligned}
& \dot{\dot{W}}=\frac{\dot{W}}{\dot{m} C_{V} T_{1}}=\left(r^{(k-1)}-1\right)\left(\frac{\alpha \eta_{E}}{r^{(k-1)}}-\frac{1}{\eta_{C}}\right), \\
& \eta=\frac{\left(r^{(k-1)}-1\right)\left(\frac{\alpha \eta_{E}}{r^{(k-1)}}-\frac{1}{\eta_{C}}\right)}{(\alpha-1)-\frac{1}{\eta_{C}}\left(r^{(k-1)}-1\right)}
\end{aligned}
$$

and

$$
\bar{P}_{\mathrm{ME}}=\frac{P_{\mathrm{ME}}}{\left(\dot{m} C_{V} T_{1} / v_{1}\right)}=\left(\frac{r^{k}-r}{r-1}\right)\left(\frac{\alpha \eta_{E}}{r^{(k-1)}}-\frac{1}{\eta_{C}}\right) .
$$

One can maximize the power output given in Eq. (14) with respect to the compression ratio, $r$, and can be found as

$$
r_{\mathrm{mp}}=\left(\alpha \eta_{C} \eta_{E}\right)^{\frac{1}{2(k-1)}} .
$$

The dimensionless maximum power output and the thermal efficiency at MP now can be found by substituting Eq. (17) into Eqs. (14) and (15) respectively as

$$
\dot{\bar{W}}_{\max }=\left(\sqrt{\alpha \eta_{C} \eta_{E}}-1\right)\left(\frac{\alpha \eta_{E}}{\sqrt{\alpha \eta_{C} \eta_{E}}}-\frac{1}{\eta_{C}}\right),
$$

and

$$
\eta_{\mathrm{mp}}=\frac{\left(\sqrt{\alpha \eta_{C} \eta_{E}}-1\right)\left(\frac{\alpha \eta_{E}}{\sqrt{\alpha \eta_{C} \eta_{E}}}-\frac{1}{\eta_{C}}\right)}{(\alpha-1)-\frac{1}{\eta_{C}}\left(\sqrt{\alpha \eta_{C} \eta_{E}}-1\right)} .
$$

In order to find the maximum thermal efficiency condition, Eq. (15) can be differentiated with respect to $r$, set the resultant derivative equal to zero (i.e. $\partial \eta / \partial r=0$ ). The optimum $r$ value is obtained as

$$
r_{\mathrm{mef}}=\left(\frac{-a_{2}-\sqrt{a_{2}^{2}-4 a_{1} a_{3}}}{2 a_{1}}\right)^{\left(\frac{1}{k-1}\right)},
$$

where

$$
\begin{aligned}
& a_{1}=\frac{\alpha\left(\eta_{E}-1\right)+1}{\eta_{C}}, \\
& a_{2}=-\frac{2 \alpha \eta_{E}}{\eta_{C}}
\end{aligned}
$$

$$
a_{3}=\alpha \eta_{E}\left(\frac{1}{\eta_{C}}+(\alpha-1)\right) .
$$

By substituting Eq. (20) into Eq. (15), the maximum thermal efficiency can be obtained as

$$
\eta_{\max }=
$$

$$
\frac{\left(\frac{-a_{2}-\sqrt{a_{2}^{2}-4 a_{1} a_{3}}}{2 a_{1}}-1\right)\left(\frac{2 a_{1} \alpha \eta_{E}}{-a_{2}-\sqrt{a_{2}^{2}-4 a_{1} a_{3}}}-\frac{1}{\eta_{C}}\right)}{(\alpha-1)-\frac{1}{\eta_{C}}\left(\frac{-a_{2}-\sqrt{a_{2}^{2}-4 a_{1} a_{3}}}{2 a_{1}}-1\right)} .
$$

It is also possible to find the optimum compression ratio at maximum mean effective pressure $\left(r_{\text {mep }}\right)$ by differentiating with respect to $r$ and seeking a maximum power density, $P_{\mathrm{ME}, \max }$, by setting

$$
\begin{aligned}
\frac{\partial \bar{P}_{\mathrm{ME}}}{\partial r} & =k r_{\mathrm{mep}}^{2(k-1)}-b_{1} r_{\mathrm{mep}}^{(k-1)}+b_{2} r-b_{3} r_{\mathrm{mep}}^{(2 k-1)} \\
+b_{4} & =0
\end{aligned}
$$

where

$$
\begin{aligned}
& b_{1}=1+\alpha \eta_{C} \eta_{E}, \\
& b_{2}=\alpha \eta_{C} \eta_{E}(k-1), \\
& b_{3}=k-1, \\
& b_{4}=\alpha \eta_{C} \eta_{E}(2-k) .
\end{aligned}
$$

The maximum dimensionless mean effective pressure, $P_{\mathrm{ME}, \max }$, and the thermal efficiency at the MEP condition, which occurs at $r=r_{\text {mep }}$, can be written respectively as

$$
\bar{P}_{\mathrm{ME}, \mathrm{max}}=\left(\frac{r_{\mathrm{mep}}^{k}-r_{\mathrm{mep}}}{r_{\mathrm{mep}}-1}\right)\left(\frac{\alpha \eta_{E}}{r_{\mathrm{mep}}^{(k-1)}}-\frac{1}{\eta_{C}}\right)
$$

and

$$
\eta_{\mathrm{mep}}=\frac{\left(r_{\mathrm{mep}}^{(k-1)}-1\right)\left(\frac{\alpha \eta_{E}}{r_{\mathrm{mep}}^{(k-1)}}-\frac{1}{\eta_{C}}\right)}{(\alpha-1)-\frac{1}{\eta_{C}}\left(r_{\mathrm{mep}}^{(k-1)}-1\right)} .
$$

\section{Results and discussion}

In terms of power, mean effective pressure and thermal efficiency a performance analysis has been carried out in order to investigate the performance of an irreversible Otto cycle. In the calculation procedure, the $k$ parameter is chosen as equal to 1.4 , and also the isentropic efficiencies for the compression and expansion processes are set to be $\eta_{C}=\eta_{E}=0.85$. The normalized power output $\left(\overline{\dot{W}} / \overline{\dot{W}}_{\max }\right)$, normalized mean effective pressure $\left(\overline{P_{\mathrm{ME}}} / \bar{P}_{\mathrm{ME}, \max }\right)$ and normalized thermal efficiency $\left(\eta / \eta_{\max }\right)$ can be plotted with respect to the compression ratio $\left(r=v_{\max } / v_{\min }\right)$ under the constraint of the second law, using Eq. (7) as shown in Fig. 2. We can observe from the figure that each objective function has a maximum for different $r$ value, which was chosen for a set of operation parameters. The optimal $r$ values at maximum $\overline{\dot{W}}$ and $\eta$ objective functions $\left(r_{\mathrm{mp}}\right.$ and $\left.r_{\mathrm{mef}}\right)$ 
can be found analytically by using Eqs. (17) and (20). The optimal $r$ value at maximum $\bar{P}_{\mathrm{ME}}$ can also be found numerically by using Eq. (22). It is obvious from Fig. 2 that the optimal compression ratios can symbolically be arranged in order as $r_{\text {mep }}<r_{\text {mp }}<r_{\text {mef }}$.

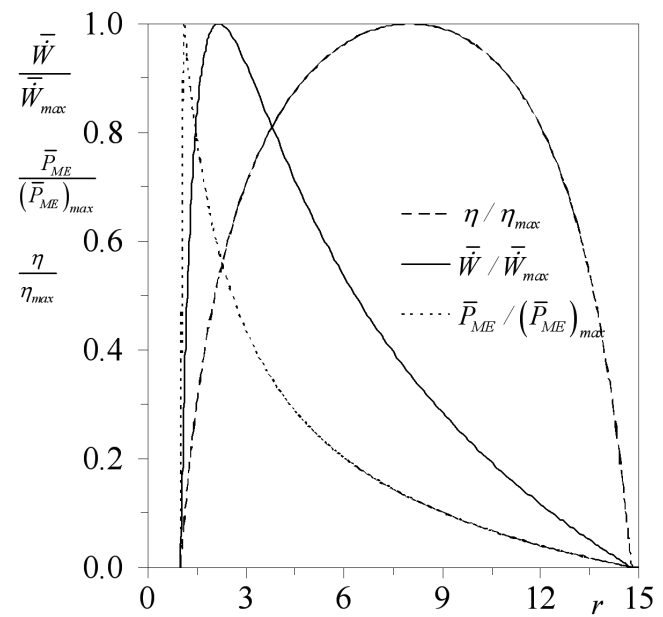

Fig. 2. The variation of the normalised power output, mean effective pressure and thermal efficiency with respect to the compression ratio for $\lambda=60$ and $\eta_{C}=$ $\eta_{E}=0.85$.

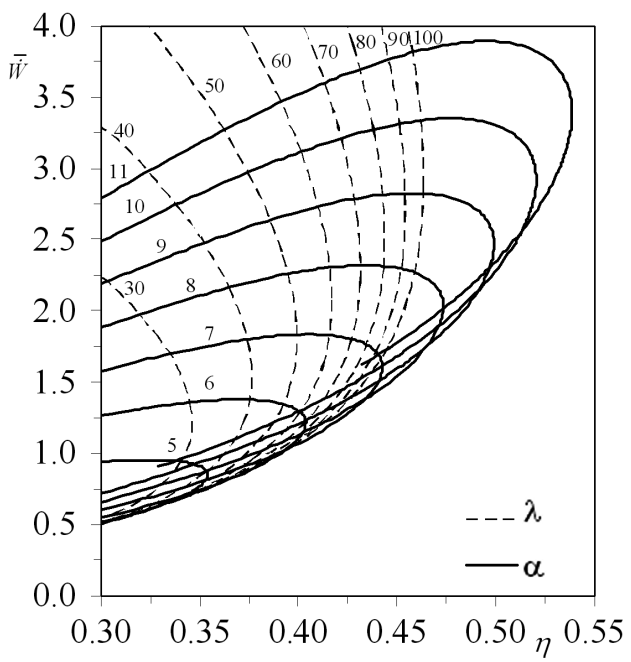

Fig. 3. The variation of the dimensionless power output with respect to the thermal efficiency for different $\alpha$ and $\lambda$ values.

Variations of the dimensionless power output $(\overline{\dot{W}})$ and dimensionless mean effective pressure $\left(\bar{P}_{\mathrm{ME}}\right)$ with respect to the thermal efficiency $(\eta)$ for different cycle temperature ratio $(\alpha)$ and cycle pressure ratio $(\lambda)$ values are presented in Figs. 3 and 4 . There is functional relation between $\alpha, \lambda$ and $r$ and this relation is given as

$$
r=\lambda / \alpha \text {. }
$$

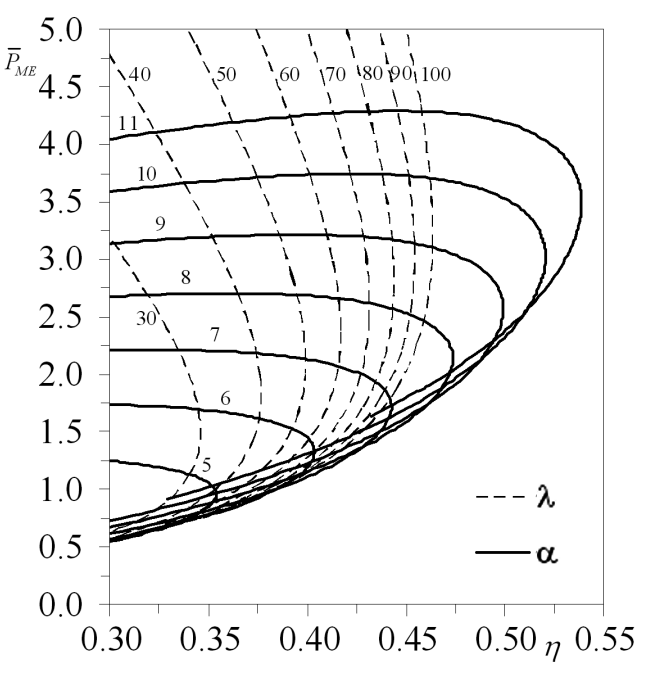

Fig. 4. The variation of the dimensionless mean effective pressure with respect to the thermal efficiency for different $\alpha$ and $\lambda$ values.

These performances given in Figs. 3 and 4 have been obtained by changing the compression ratio $(r)$ for a given value of $\alpha$ or $\lambda$ using the relationship given in Eq. (25). From these figures, we can evaluate the effects of the design parameters on the power output, mean effective pressure and thermal efficiency for better performance. The curves in Figs. 3 and 4 are all in loop-like shape and thus they have two different maxima for both axes, which are $\overline{\dot{W}}-\eta$ and $\bar{P}_{\mathrm{ME}}-\eta$, respectively. By observing these figures, it can be observed that, as $\alpha$ and $\lambda$ increase the general and optimal performances in terms of $\overline{\dot{W}}, \bar{P}_{\mathrm{ME}}$ and $\eta$ increase significantly. The effects of $\alpha$ and $\lambda$ on the power output, thermal efficiency and mean effective pressure are seen clearly by observing Figs. 3 and 4. It is observed from these figures that there are optimum values of $\alpha$ and $\lambda$ in terms of these performance criteria. These optimal values can be determined realistically with a thermo-economic optimization study by considering the relations of $\alpha$ and $\lambda$ with the costs and engine sizes. This study may offer a new insight into the thermo-economic optimization of real Otto cycles.

\section{Conclusions}

A comparative performance analysis based on MEP, $\mathrm{MP}$ and MEF criteria has been performed for an irreversible Otto heat-engine model, consisting one constant volume heating process, one constant volume cooling process and two adiabatic processes with consideration of internal irreversibilities. In this perspective, values of the design parameters that maximize the mean effective pressure, power output and thermal efficiency of the irreversible Otto cycle have been investigated. The relations between the mean effective pressure and the compression ratio, and between the power output and the compression ratio, and between the thermal efficiency and the 
compression ratio of the cycle have been derived. The mean effective pressure versus thermal efficiency curves and the power output versus thermal efficiency curves for the Otto cycle have been illustrated for different cycle temperature ratios and cycle pressure ratios. The results presented in this analysis may provide guidelines for determination of the optimal design and operating conditions of real Otto cycles.

\section{References}

[1] A. Bejan, J. Appl. Phys. 79, 1191 (1996).

[2] L. Chen, C. Wu, F. Sun, J. Non-Equilib. Thermodyn. 24, 327 (1999).

[3] L. Chen, F. Sun, Advances in Finite-Time Thermodynamics: Analysis and Optimization, Nova Sci. Pub., New York 2004.

[4] F.L. Curzon, B. Ahlborn, Am. J. Phys. 43, 22 (1975).

[5] B. Andresen, P. Salamon, R.S. Berry, Phys. Today 37, 62 (1984).

[6] S. Sieniutycz, P. Salamon, Advances in Thermodynamics: Finite-Time Thermodynamics and Thermoeconomics, Taylor and Francis, New York 1990.

[7] A. Durmayaz, O.S. Sogut, B. Sahin, H. Yavuz, Prog. Ener. Comb. Sci. 30, 175 (2004).

[8] M. Mozurkewich, R.S. Berry, J. Appl. Phys. 53, 34 (1982).

[9] S.A. Klein, Trans. ASME J. Eng. Gas Turbine Pow. 113, 511 (1991).
[10] C. Wu, D.A. Blank, J. Inst. Energy 65, 86 (1992).

[11] C. Wu, D.A. Blank, Energy Convers. Manag. 34, 1255 (1993).

[12] A.C. Hernandez, A. Medina, J.M.M. Roco, S. Velasco, Eur. J. Phys. 16, 73 (1995).

[13] F. Angulo-Brown, J.A. Rocha-Martinez, T.D. Navarrete-Gonzalez, J. Phys. D, Appl. Phys. 29, 80 (1996).

[14] L. Chen, F. Sun, C. Wu, Energy Convers. Manag. 39, 643 (1998).

[15] Y. Ge, L. Chen, F. Sun, C. Wu, Int. J. Therm. Sci. 44, 506 (2005).

[16] Y. Ge, L. Chen, F. Sun, C. Wu, Int. J. Exergy 2, 274 (2005).

[17] O.A. Ozsoysal, Energy Convers. Manag. 47, 1051 (2006).

[18] J. Chen, Y. Zhao, J. He, Appl. Energy 83, 228 (2006).

[19] S.S. Hou, Energy Convers. Manag. 48, 1683 (2007).

[20] Y. Ge, L. Chen, F. Sun, C. Wu, Appl. Energy 85, 618 (2008).

[21] J.C. Lin, S.S. Hou, Energy Convers. Manag. 49, 1218 (2008).

[22] M. Gumus, M. Atmaca, T. Yilmaz, Int. J. Energy Res. 33, 745 (2009).

[23] R. Ebrahimi, Acta Phys. Pol. A 117, 887 (2010). 